\title{
ATLAS Inner Detector reconstruction and analysis with data from the combined test beam
}

\author{
Sergio González-Sevilla*† \\ Instituto de Física Corpuscular (IFIC), València, Spain \\ E-mail: segonzaleific.uv.es
}

\section{Maria Jose Costa}

CERN, Switzerland

\section{Wolfgang Liebig}

CERN, Switzerland

\section{Salvador Marti i Garcia}

Instituto de Física Corpuscular (IFIC), València, Spain

\section{Andreas Salzburger}

CERN, Switzerland

The full ATLAS Inner Detector reconstruction chain has been tested for the first time with real data from the 2004 ATLAS combined testbeam. A total of 22 million validated events were collected using the final readout electronics and DAQ system with different particle beams with varying different energies and magnetic field values.

This data allowed to test and develop the new track reconstruction software. The underlying event data model and set of interfaces for algorithms provide a very high level of commonality across detector configurations, such as the ATLAS selection run in the combined testbeam. This reconstruction makes use of a new extrapolation package which implements different propagation methods (straight line or helical track models as well as Runge-Kutta integrational method) with and without continous material interaction.

New features had to be added to the software in order to deal with real data as improvements in the detector description including possible inperfections and introducing calibration and alignment corrections which also meant using for the first time the conditions database designed for ATLAS. Monitoring algorithms were also developed and tested during the data taking.

The performance of each subystem (Pixel, SCT and TRT) and of the whole Inner Detector was studied in detail. A full simulation was also produced for the testbeam layout using the same tools and algorithms developed for the real ATLAS experiment. A comparison of real and simulated data allowed then to tune the simulation and digitization processes.

International Europhysics Conference on High Energy Physics

July 21 st - 27th 2005

Lisboa, Portugal

\footnotetext{
${ }^{*}$ Speaker.

On behalf of the ATLAS Inner Detector Software Group
} 


\section{Introduction}

The ATLAS experiment is a general purpose detector designed to fully exploit the physics potential of the Large Hadron Collider (LHC), the proton-proton collider actually under installation at CERN. ATLAS is formed by three sub-detector systems: the Inner Detector, the Calorimetry system and the Muon Spectrometer. In the Combined Testbeam (CTB), a full barrel slice of the ATLAS detector, starting from the interaction point at the pseudorapidity value $\eta=0$, was tested at the CERN H8 testbeam facility during 2004. The final combined setup included detectors from all the different ATLAS subsystems, and the main goal was the pre-commissioning of almost the final elements of the experiment and the study of the detector performance in a realistic data taking environment.

\section{Inner Detector setup}

The Inner Detector (ID) layout in the CTB was composed by 6 Pixel modules, arranged in three layers, 8 SCT modules in four layers and 6 TRT modules. Both the pixel and SCT detectors were located inside a magnet delivering a magnetic field up to $1.4 \mathrm{~T}$; the TRT modules were out of the magnet. The distances between detectors along the beam axis and the overlap between modules within the same layer were chosen to be as close as possible as the corresponding designed values in ATLAS.

A complete physics program was undertaken during the period in which the three subdetectors were collecting data in a combined mode. The CERN Super Proton Synchrotron (SPS) provides secondary beams to the $\mathrm{H} 8$ testing area. A highly variated number of runs were recorded, reflecting different kind of particle beams including electrons, pions, muons and photons, with energies varying from $2 \mathrm{GeV} / \mathrm{c}$ up to $180 \mathrm{GeV} / \mathrm{c}$. The magnetic field and the polarity of the current at the coils was also varied. In addition, a dedicated period of data taking was devoted for runs with extra material between the different detectors, in order to mimic the services of the ID at $\eta=1.6$, allowing to perform multiple scattering studies. Therefore, some aluminum plates were inserted between the Pixels and SCT $\left(11 \% X_{0}\right)$, and between the SCT and the TRT $\left(22 \% X_{0}\right)$.

At the end of the data taking, a total of 22 million events were validated by the ID offline community as usable for further physics analysis.

\section{Offline Reconstruction Chain}

The CTB represented a major challenge from the software side, as the same reconstruction chain that will be used in the real experiment had to be commissioned. The offline reconstruction chain was implemented within the context of the new ATLAS Event Data Model (EDM). The EDM, developed under the Athena software framework, is designed to provide common tools to different parts of the ATLAS detector, sharing similar requirements in terms for example of input data objects or processing stages.

The full offline reconstruction chain is schematized in figure 1. Its flexible and modular design allows to take as input either real data coming from the detectors or GEANT4 simulated events. In both cases, the detector description is performed using the same ensemble of geometrical primitives (GeoModel) than used for modeling the whole ATLAS detector. Different track fitters 
(xKalman, CTBTRacking) and track re-fitters (Kalman fitter, distributed Kalman fitter) were available for their use in the CTB, delivering reconstructed tracks by the ID. Within the EDM, the track object is defined by means of a $\mathrm{C}++$ class, common to the muon system, so that combined track reconstruction using the ID and the muon detectors can be performed. The final output suitable for physics analysis is available in several formats, such as Event Summary Data (ESD) or combined ntuples (CBNT).

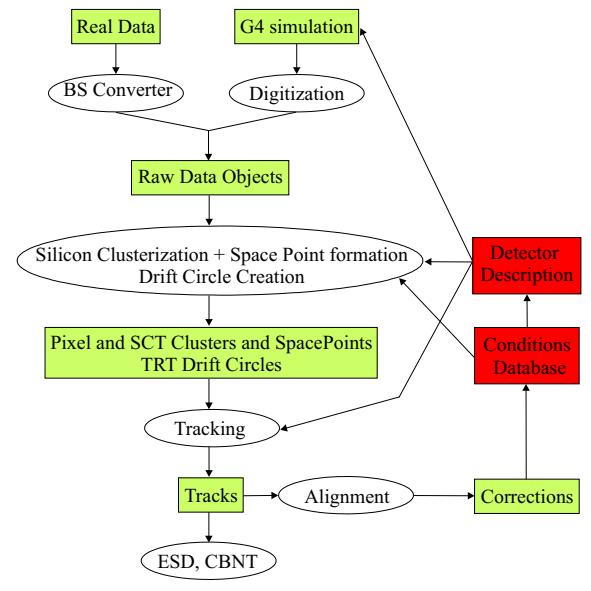

Figure 1: Offline Reconstruction chain.

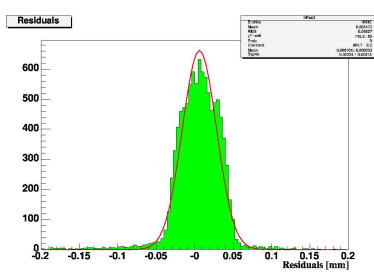

(a)

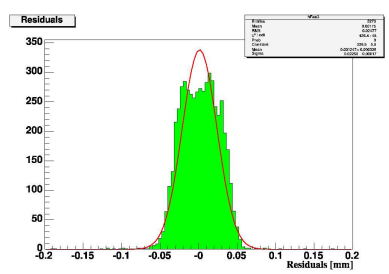

(b)
Figure 2: Comparison of residuals obtained in one plane of a SCT module for a $180 \mathrm{GeV}$ pion beam for data (a) and simulation with GEANT4 (b).

\section{Alignment and calibration}

Different sub-periods of data taking were identified as corresponding to detector movements in the setup. The alignment is performed in two steps. First, all the silicon modules (Pixels and SCT) are aligned following an iterative procedure in which residuals with respect to the track intersection at each plane are minimized (see figure 2). Second, the TRT is aligned with respect the silicon modules by extrapolating tracks reconstructed using just the information coming from the Pixel and SCT detectors.

In ATLAS, the calibration and alignment algorithms will produce "non-event" data that will be stored in the conditions database. For the first time during the CTB, the complete interaction with the conditions database was possible by means of the development of dedicated algorithms and tools, which allowed the storage for example of calibration constants such as dead and noisy channels, $R$ - $t$ relations for the TRT individual straw tubes, and alignment corrections for all three subdetectors.

\section{Results}

For the first time, the full offline reconstruction chain presented in section 3 was used in order to fit tracks using the three ID subdetectors (see figure 3(a)). An example of reconstructed momentum distribution is shown in figure 3(b). The individual detector performance was also tested. An example is shown in figure 3(c), where the TRT capabilities for electron identification are studied, allowing to perform a fine tuning of the simulation afterwards. 
Among the study of the performance of the three subdetectors and the tracking capabilities of the whole ID system, more ambitious objectives are also in mind, as for example performing combined studies with other ATLAS subdetectors such as the calorimeters or the muon system. This is shown on figure 3(d), where a extrapolated track from the ID to the Liquid Argon Calorimeter allows to distinguish between primary electrons and photon conversions produced by material interactions.

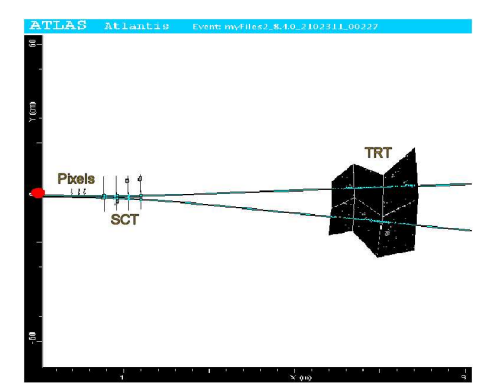

(a)

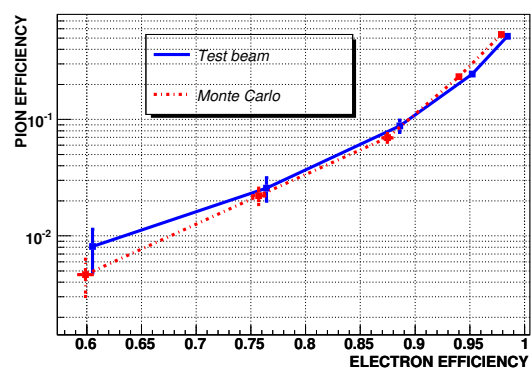

(c)

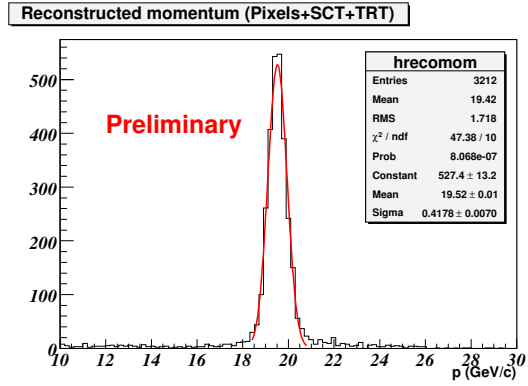

(b)

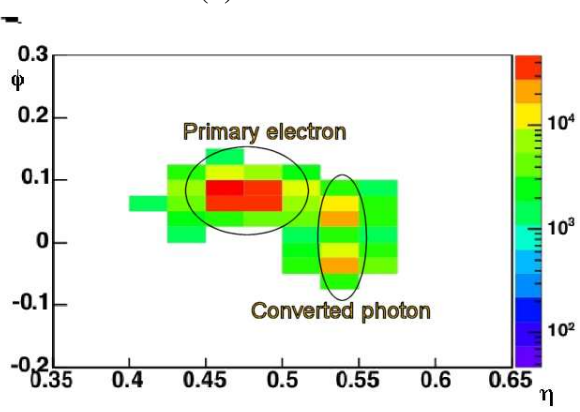

(d)

Figure 3: (a) Reconstructed tracks for a $20 \mathrm{GeV} / \mathrm{c}$ pion beam with a $1.4 \mathrm{~T}$ magnetic field from left to right, through the Pixel, SCT and TRT detectors. (b) Reconstructed momentum of a $20 \mathrm{GeV} / \mathrm{c}$ pion beam using the three ID subdetectors. (c) Rejection curves for for electrons and pions using the TRT detector comparing real and simulated data for a very low energy beam $(2 \mathrm{GeV} / \mathrm{c})$. (d) Primary electron and photon conversions distinction in the cells of the LAr calorimeter by extrapolating tracks reconstructed with the ID.

\section{Conclusions and Acknowledgments}

The ATLAS 2004 Combined Testbeam was a success for the Inner Detector community. For the first time, a common data taking was possible between all the different subdetectors using the final offline reconstruction chain.

\section{Acknowledgments}

The work presented here is the result of the dedicated efforts of both the Inner Detector software group and of the hardware experts during the ATLAS 2004 CTB. 